\title{
Resistenza ai fluorochinoloni in Escherichia coli isolati da infezioni delle vie urinarie (IVU) in pazienti ospedalizzati in unità di terapia intensiva (UTI).
}

\author{
Paola Pistarà, Stefania Mannelli, Anna Marchese, Simona Roveta. \\ Università di Genova, DISCAT - Sezione di Microbiologia, Largo R. Benzi 10, I6I32 Genova.
}

Key words: uropathogenic Escherichia coli, fluoroquinolones resistance, ciprofloxacin, norfloxacin, pipemidic acid.

Fluoroquinolones-resistance among Escherichia coli strains isolated from urinary tract infections (UTI) of patients admitted into intensive care units (ICU)

\section{SUMMARY}

Background: Fluoroquinolones are an important class of antibiotics for the treatment of urinary tract infections that have axcellent activity against Escherichia coli, one of the most frequently encountered pathogens. Several European studies have reported an increase of resistance to quinolones among uropathogenic $E$. coli. We conducted this study to update our knowledge on this evolution.

Materials and methods: We evaluated the resistance phenotype of 203 clinical strains of $E$. coli collected from urine specimens. The following antimicrobial agents were tested: ampicillin, amoxiciclin-clavulanate, piperacillintazobactam, cefamandole, cefotaxime, ceftazidime, cefepime, aztreonam, imipenem, trimethoprimsulfamethoxazole, gentamicin, amikacin, ciprofloxacin, pipemidic acid, norfloxacin, nitrofurantoin. Disk diffusion tests were carried out as suggested by the CLSI (2006); strains were assigned to the susceptibility categories (susceptible, intermediate and resistant) interpreting results according to the established breakpoints.

Results: Resistance to quinolones (pipemidic acid, norfloxacin and ciprofloxacin) was about of $45 \%$ and resistance to ampicillin, trimethoprim-sulfamethoxazole was $57.1 \%$ and $55.2 \%$, respectively. Resistance rates less than $5 \%$ was found for cefepime, amikacin and imipenem.

Conclusions: This study confirms the evolution through resistance to quinolones of uropathogenic E. coli isolates. The selective pressure exerted by fluoroquinolones may influence this evolution. Therapeutic alternatives, surveillance, and restriction of fluoroquinolones use are needed to control this spread of resistance.

\section{INTRODUZIONE}

Le infezioni delle vie urinarie (IVU) costituiscono globalmente nella popolazione la causa più comune di infezione batterica e rappresentano il $30-40 \%$ delle infezioni ospedaliere, essendo la forma più frequente di infezione nosocomiale $(5$, 9, 11). Le IVU di origine nosocomiale sono molto spesso infezioni complicate e la loro eziologia differisce notevolmente da quella delle infezioni comunitarie non complicate coinvolgendo una eterogenea gamma di specie, sia Gram-negative che Gram-positive. Sebbene l'eziologia possa variare nel tempo e in funzione della località geografica, il patogeno più frequentemente isolato rimane solitamente l'Escherichia coli. Per anni i farmaci di prima scelta impiegati in queste patologie sono stati amoxicillina, co-trimoxazolo, nitrofurantoina e fluorochinoloni (norfloxacina e ciprofloxacina). Dopo il tramonto dell'impiego delle aminopenicilline dovuto alla diffusione di beta-lattamasi, il co-trimoxazolo ha goduto di larga preferenza sino alla metà degli anni Novanta, quando una rapida disseminazione della refrattarietà a questo chemioterapico ne ha ridotto in maniera considerevole la sensibilità in $E$. coli $(20,24)$. Così come l'utilizzo di ß-lattamici e cotrimossazolo nella terapia di prima linea delle IVU diede luogo ai primi fenomeni di diffusione della resistenza a queste molecole, lo stesso fenomeno viene segnalato negli ultimi anni in vari paesi anche per i fluorochinoloni, che sono i farnaci maggiormente utilizzati nel trattamento delle IVU (1, 6-7, 10, 12-17, 25-26, 29).

Questo studio si propone di verificare se l'E. coli uropatogeno sta acquisendo resistenza a questa famiglia di chemioterapici anche nella nostra realtà locale, con particolare riferimento ai ceppi isolati da pazienti ospedalizzati nelle unità di terapia intensiva. È stata, pertanto, valutata in stipiti di $E$. coli uropatogeni recentemente isolati nei suddetti reparti la sensibilità alle varie classi di chemioterapici indagando, in particolare, la percentuale di sensibilità nei confronti di tre fluorochinoloni: ciprofloxacina, norfloxacina e acido pipemidico.

\section{MATERIALI E METODI}

Presso la Sezione di Microbiologia dell'Università di Genova, nel periodo febbraio 
2005 - gennaio 2006, sono state raccolte 2256 urinocolture provenienti da pazienti con infezioni complicate del tratto urinario ricoverati in unità di terapia intensiva (Anestesia e Rianimazione; Nefrologia, Dialisi e Trapianti; Clinica Neurochirurgia e Neurotraumatologica).

La carica batterica del campione è stata valutata con il Sistema Uro-Quick, un metodo di screening rapido che permette anche la determinazione del potere antibatterico residuo (P.A.R test) per accertare che un risultato negativo non sia dovuto ad esso. In contemporanea all'esame colturale, è stato eseguito un esame microscopico del campione dopo colorazione di Gram per verificare la presenza di piuria ed ematuria, indicatori di infezione in corso.

I campioni risultati positivi allo screening sono stati seminati sia in terreno arricchito (Columbia agar sangue $5 \%$ ), per permettere la crescita di microrganismi Gram-positivi e Gram-negativi, sia in terreno selettivo (Mac-Conkey agar) per favorire l'isolamento dei soli Gram-negativi. Le piastre sono state quindi incubate in aerobiosi a $37^{\circ} \mathrm{C}$ per $18-24$ ore.

L'identificazione dei germi è avvenuta tramite l'impiego di prove metaboliche e biochimiche. Nello studio sono state prese in considerazione infezioni monomicrobiche e polimicrobiche sostenute da $E$. coli.

L'antibiogramma è stato eseguito con il metodo della diffusione su disco (Kirby-Bauer), utilizzando come terreno il Muller-Hinton agar inoculato con la sospensione ( $0.5 \mathrm{McF}$ arland) del ceppo in esame e incubato a $37^{\circ} \mathrm{C}$ per 24 ore. Sono stati saggiati i seguenti antibiotici: ampicillina (AMP), amoxiciclina/acido clavulanico (AMC), piperacillina/tazobactam (TZP), cefamandolo (MA), cefotaxime (CTX), ceftazidime (CAZ), cefepime (FEP), aztreonam (ATM), imipenem (IPM), trimetropim/sulfametazolo (STX), gentamicina (GN), amikacina (AK), ciprofloxacina (CIP), acido pipemidico (PIP), norfloxacina (NOR), nitrofurantoina $(\mathrm{F})$. I risultati sono stati valutati in base alle indicazioni riportate da CSLI (2005) (2).

\section{RISULTATI}

Delle 2256 urinocolture analizzate, 492 sono risultate positive $(22 \%)$. Gli stipiti di E. coli isolati sono stati 203 , pari al $41.3 \%$ dei campioni positivi e al $10 \%$ di tutte le urinocolture: tra questi il $7.4 \%$ proveniva da campioni monomicrobici, mentre il $2.6 \%$ da infezioni polimicrobiche.

Il $60 \%$ dei pazienti in cui è stata evidenziata la presenza di $E$. coli nelle urine era di sesso femminile, il $40 \%$ maschile.

Nella Figura I sono rappresentate le percentuali di resistenza riscontrate relativamente ai vari anti- biotici, mentre nella Tabella 1 sono riportate in maniera più dettagliata le percentuali di antibiotico-sensibilità. Le resistenze a AMP e STX erano superiori al 55\%, quelle ai tre fluorochinoloni (CIP, PIP, NOR) sono risultate in media del $45 \%$. Nell'ambito di quest'ultima classe di farmaci, CIP e NOR (55.1\% di ceppi sensibili) si sono dimostrate leggermente più attive dell'acido pipemidico (53.7\% di stipiti sensibili). Livelli di resistenza minori del $10 \%$ sono stati riscontrati nelle cefalosporine di terza generazione come ceftazidime e cefepime (rispettivamente 92.1 e $94.2 \%$ di ceppi sensibili). Percentuali di resistenza inferiori al $5 \%$ sono stati evidenziati per AK (1\%), carbapenemici $(1.4 \%)$, TZP $(2.5 \%)$ e nitrofurantoina (3.4\%).

Tra i ceppi che presentavano assenza si sensibilità ai fluorochinoloni è stata verificata l'eventuale presenza di multi-resistenza: nel $9.8 \%$ è stata riscontrata resistenza contemporanea ad altre due classi di antibiotici e nel 5.8\% ad altre tre classi di farmaci oltre a quella dei fluorochinoloni, per un totale del $15.6 \%$ di ceppi multi-resistenti.

La presenza di potere antimicrobico residuo (P.A.R. positivo) è stata segnalata nell' $8.3 \%$ delle urine positive per E. coli.

Tabella I: Percentuali di antibiotico-sensibilità di 203 stipiti di E. coli

\begin{tabular}{lccc}
\hline Antibiotici & $\mathbf{S}$ & $\mathbf{I}$ & $\mathbf{R}$ \\
\hline AMP & 42.9 & 0 & 57.1 \\
\hline AMC & 76.4 & 12.8 & 10.8 \\
\hline TZP & 97.5 & 0 & 2.5 \\
\hline MA & 78.7 & 3.0 & 18.2 \\
\hline CTX & 78.3 & 4.5 & 17.2 \\
\hline CAZ & 92.1 & 1.5 & 6.4 \\
\hline FEP & 94.2 & 1.4 & 4.4 \\
\hline ATM & 78.8 & 3.0 & 18.2 \\
\hline IPM & 98.6 & 0 & 1.4 \\
\hline STX & 42.4 & 2.4 & 55.2 \\
\hline GN & 84.7 & 0 & 15.3 \\
\hline AK & 99.0 & 0 & 1.0 \\
\hline CIP & 55.1 & 0 & 44.9 \\
\hline PIP & 53.7 & 0.5 & 45.8 \\
\hline NOR & 55.1 & 0 & 44.9 \\
\hline F & 96.6 & 0 & 3.4 \\
\hline
\end{tabular}

\section{CONCLUSIONI}

Nel presente studio è stata evidenziata una presenza significativa di E. coli (oltre il 40\%) nei campioni d'urina positivi, con una percentuale più elevata in quelli provenienti da pazienti di sesso femminile. Per quanto riguarda la sensibilità di questo patogeno nei confronti dei fluorochinoloni, i risultati riscontrati indicano una preoccupante diffusione della resistenza a questa classe 


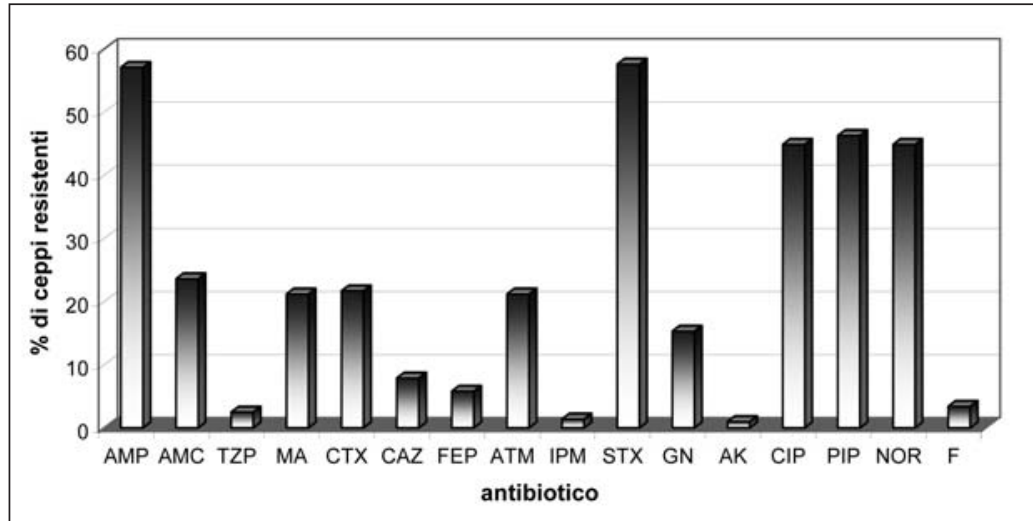

Figura I. Percentuali di resistenza agli antibiotici di 203 stipiti di E. coli.

Legenda: ampicillina (AMP), amoxiciclinalacido clavulanico (AMC), piperacillina/tazobactam (TZP), cefamandolo (MA), cefotaxime (CTX), ceftazidime (CAZ), cefepime (FEP), aztreonam (ATM), imipenem (IPM), trimetropim/sulfametazolo (STX), gentamicina $(G N)$, amikacina (AK), ciprofloxacina (CIP), acido pipemidico (PIP), norfloxacina (NOR), nitrofurantoina $(F)$.

$S$, sensibile; I, intermedio; $R$, resistente.

\section{di farmaci.}

Gli antibiotici che, in questo studio, mantengono ancora percentuali di sensibilità in vitro sufficientemente elevate sono: le cefalosporine di terza generazione, come cefepime e ceftazidime, l'amminoglicoside amikacina, il carbapenemico imipenem, la nitrofurantoina e il B-lattamico coniugato all'inibitore suicida piperacillina-tazobactam.

Le infezioni complicate del tratto urinario sostenute dai ceppi di E. coli stanno, dunque, diventando (soprattutto in ambito nosocomiale) un problema sempre più serio: da un lato vi è la problematica della diffusione di resistenze nei confronti di diversi antibiotici, compresi i fluorochinoloni, utilizzati per la loro efficacia antibatterica come farmaci di prima linea contro le IVU per parecchi anni, e dall'altro i vari fattori di patogenicità di cui è dotato questo microrganismo. Questi fattori, specialmente in caso di concomitante diminuzione delle difese immunitarie dell'ospite, possono permettere ad $E$. coli la progressione verso infezioni di notevole gravità come pieolonefriti, infezioni delle vie respiratorie, sepsi, peritoniti e artriti $(3,19,22-23)$.

La refrattarietà di $E$. coli ai fluorochinoloni assume significato e gravità ancora maggiore considerando che in una significativa percentuale di casi essa risulta associata anche a quella verso altre classi di antibiotici. I pazienti ospedalizzati, infatti, vengono spesso sottoposti a ripetuti cicli di terapia antimicrobica, pertanto l'isolamento dalle urine di ceppi dotati di resistenze, anche multiple, non rappresenta un evento raro $(4,8,18,21)$. Anche questo studio conferma un'esposizione elevata dei pazienti a farmaci antibiotici, testimo- niata dalla presenza di una significativa percentuale $(15.6 \%)$ di ceppi multi-resistenti.

Occorre, dunque, sottolineare la fondamentale importanza di formulare un'adeguata terapia farmacologia; sia per conseguire una rapida ed efficace risposta terapeutica, sia per prevenire la selezione e la diffusione dell'antibiotico resistenza nei microrganismi. (27-31).

L'utilizzo di una terapia empirica è solitamente sconsigliato, un approccio corretto prevede l'esecuzione dell'urinocoltura con il relativo antibiogramma. In particolare, l'utilizzo dei fluorochinoloni dovrebbe essere limitato ai soli casi in cui è stata riscontrata la sensibilità del ceppo dopo l'esecuzione dell'antibiogramma. In caso di infezioni nosocomiali gravi, quando l'urgenza clinica non consente di mirare la terapia, possono essere impiegate molecole come le cefalosporine di terza generazione, i carbapenemici e gli amminoglicosidi.

\section{BIBLIOGRAFIA}

1. Barisic Z, Borzic E, Kraljevic KS, Carev M, Zoranic $\mathrm{V}$, Kaliterna V. Rise in ciprofloxacin resistance in Escherichia coli from urinary tract infections from 1999-2004. Int J Antimicrob Agents. 2005; 25: 550551.

2. Clinical and Laboratory Standards Institute/NCCLS. Performance Standards for Antimicrobial susceptibility testing; fifteenth International Supplement. CLSI/NCCLS document M100-S15. Clinical and Laboratory Standards Institute, Wayne, Pennsylvania, 2005.

3. Emody L, Kerenyi M, Nagy G. Virulence factors of uropathogenic Escherichia coli. Int J Antimicrob Agents. 2003; 22 (Suppl 2): 29-33.

4. Farrell DJ, Morrissey I, De Rubeis D., Robbins M., Felmingham D. A UK multicentre study of the antimicrobial susceptibility of bacterial pathogens causing urinary tract infection. 2003. J Infect. 46: 94-100.

5. Gauzit R, Lepape A, Moine P. Nosocomial urinary tract infection. Ann Fr Anesth Reanim. 2004; 23: 3-5.

6. Goettsch W, van Pelt W, Nagelkerke $\mathrm{N}$, et al. Increasing resistance to fluoroquinolones in Escherichia coli from urinary tract infections in the netherlands. J Antimicrob Chemother. 2000; 46: 223 228.

7. Gomez-Martinez J, Marco F, Mensa J, Espasa M, Martinez JA, Jimenez de Anta MT. In vitro activity of fluoroquinolones and oral beta lactam antibiotics against clinical isolates of Escherichia coli. Rev Esp Quimioter. 1999; 12: 54-57.

8. Gordon KA, Jones RN, SENTRY Participant Groups (Europe, Latin America, North America). 
Susceptibility patterns of orally administered antimicrobials among urinary tract infection pathogens from hospitalized patient in North America: comparison report to Europe and Latin America. Results from the SENTRY Antimicrobial Surveillance Program (2000). Diagn Microbiol Infect Dis. 2003; 45: 295301.

9. Hashmi S, Kelly E, Rogers SO, Gates J. Urinary tract infection in surgical patients. Am J Surg. 2003; 186: 53-56.

10. Junquera S, Loza E, Baquero F. Changes in the antimicrobial susceptibility of Escherichia coli isolates from nosocomial versus community-acquired urinary tract infections. Enferm Infecc Microbiol Clin. 2005; 23: 197-201.

11. Kalsi J, Arya M, Wilson P, Mundy A. Hospitalacquired urinary tract infection. Int J Clin Pract. 2003; 57: 388-391.

12. Karaca Y, Coplu N, Gozalan A, Oncul O, Citil BE, Esen B. Co-trimoxazole and quinolone resistance in Escherichia coli isolated from urinary tract infections over the last 10 years. Int J Antimicrob Agents. 2005; 26: $75-77$.

13. Karlowsky JA, Hoban DJ, Decorby MR, Laing NM, Zhanel GG. Fluoroquinolone-resistant urinary isolates of Escherichia coli from outpatients are frequently multidrug resistant: results from the North American Urinary Tract Infection Collaborative Alliance-Quinolone Resistance study. Antimicrob Agents Chemother. 2006; 50: 2251-2254.

14. Lemort ML, Neuville S, Medus M, et al. Comparative susceptibility evolution in Escherichia coli from urinary tract infections in outpatients and inpatients at Perpignan hospital in 2002 and 2004. Pathol Biol (Paris). 2006, in stampa.

15. Mahamat A, Daures JP, Sotto A. Evaluation of the relation between consumption of fluoroquinolones and emergence of resistance among Escherichia coli: contribution of observational and quasi-experimental studies. Med Mal Infect. 2005; 35: 543-548.

16. Mahamat A, Lavigne JP, Fabbro-Peray P, Kinowski JM, Daures JP, Sotto A. Evolution of fluoroquinolone resistance among Escherichia coli urinary tract isolates from a French university hospital: application of the dynamic regression model. Clin Microbiol Infect. 2005; 11: 301-306.

17. McDonald LC, Chen FJ, Lo HJ, et al. Emergence of reduced susceptibility and resistance to fluoroquinolones in Escherichia coli in Taiwan and contributions of distinct selective pressures. Antimicrob Agents Chemother. 2001; 45: 3084-3091.

18. Muratani T, Matsumoto T. Urinary tract infection caused by fluoroquinolone - and cephem-resistant Enterobacteriaceae. Int J Antimicrob Agents. 2006; 28 (Suppl 1): S10-S13.

19. Nagy G, Altenhoefer A, Knapp O, et al. Both alphahaemolysin determinants contribute to full virulence of uropathogenic Escherichia coli strain 536. Microbes Infect. 2006; 8: 2006-20012.

20. Nicolle LE. Urinary tract infection: traditional pharmacologic therapies. Am J Med 2002; 113 (Suppl. 1A): S 35- S 44.

21. Ozden M, Kalkan A, Demirdag K, Kilic SS, Ozdarendeli A. Ciprofloxacin and co-trimoxazole resistance and extended spectrum beta-lactamase production in Escherichia coli strains isolated from urinary tract infections. Int J Antimicrob Agents. 2003; 21: 492-493.

22. Schneider G, Dobrindt U, Bruggemann H, et al. The pathogenicity island-associated K15 capsule determi- nant exhibits a novel genetic structure and correlates with virulence in uropathogenic Escherichia coli strain 536. Infect Immun. 2004; 72: 5993-6001.

23. Sotto A, De Boever CM, Fabbro-Peray P, Gouby A, Sirot D, Jourdan J. Risk factors for antibiotic-resistant Escherichia coli isolated from hospitalized patients with urinary tract infections: a prospective study. J Clin Microbiol. 2001; 39: 438-444.

24. Stamm WE, Norrby SR. Urinary tract infections: disease panorama and challenges. J Infect Dis 2001;183 (Suppl.1):S1-S4.

25. Talon D, Lallemand-De-Conto S, Thouverez M, Bertrand X. E. coli: resistance to quinolones and betalactams of clinical strains isolated in the FrancheComte region of France. Pathol Biol (Paris). 2004; 52: 76-81.

26. Wagenlehner F, Stower-Hoffmann J, SchneiderBrachert W, Naber KG, Lehn N. Influence of a prophylactic single dose of ciprofloxacin on the level of resistance of Escherichia coli to fluoroquinolones in urology. Int J Antimicrob Agents. 2000; 15: 207-211.

27. Wagenlehner FM, Naber KG. Current challenges in the treatment of complicated urinary tract infections and prostatitis. Clin Microbiol Infect. 2006; 12 (Suppl 3): $67-80$.

28. Wagenlehner FM, Naber KG. Treatment of bacterial urinary tract infections: presence and future. Eur Urol. 2006; 49: 235-44.

29. Wagenlehner FM, Niemetz A, Dalhoff A, Naber KG. Spectrum and antibiotic resistance of uropathogens from hospitalized patients with urinary tract infections: 1994-2000. Int J Antimicrob Agents. 2002; 19: 557-564.

30. Wagenlehner FM, Weidner W, Naber KG. Emerging drugs for bacterial urinary tract infections. Expert Opin Emerg Drugs. 2005; 10: 275-298.

31. Wagenlehner FM, Weidner W, Naber KG. Emergence of antibiotic resistance amongst hospital-acquired urinary tract infections and pharmacokinetic/pharmacodynamic considerations. J Hosp Infect. 2005; 60: 191200 .

\section{Simona Roveta}

Sez. Microbiologia - DISCAT - Università degli Studi di Genova Largo Rosanna Benzi 10 - 16132 Genova Tel.: 010-3537655 - Fax: 010-3537698 E-mail: simona.roveta@unige.it 